

\title{
The structure of uniaxially stretched isotactic polypropylene sheets: imaging with frequency-modulation atomic force microscopy
}

Kiminori Uchida ${ }^{a}$, Kazuki Mita ${ }^{a}$, Osamu Matsuoka ${ }^{a}$, Takeharu Isaki ${ }^{a}$, Kenjiro Kimurab and Hiroshi Onishi ${ }^{*}$

a Advancing Analysis Laboratory, Mitsui Chemicals, Inc., 580-32 Nagaura, Sodegaura, Chiba 299-0265, Japan

${ }^{\mathrm{b}}$ Department of Chemistry, Graduated School of Science, Kobe University, Rokko-dai, Nada, Kobe, 657-8501, Japan

* Corresponding author. E-mail: oni@kobe-u.ac.jp (H. Onishi).

\begin{abstract}
Isotactic polypropylene sheets were uniaxially stretched and observed with a frequency-modulation atomic force microscope operated in phenyloctane liquid. Crystalline lamellae were seen in fibrils with their axis parallel to the stretched direction. Individual $\mathrm{CH}_{3}$ side-chains of three-fold helices were identified in the lamellae. Fragmentation of the lamellae was induced by further stretching. The real-space features observed with the microscope were successfully compared with X-ray scattering results obtained in a synchrotron radiation facility.
\end{abstract}

Key Words: Frequency-Modulation Atomic Force Microscopy, polypropylene, X-ray scattering, uniaxial stretching 


\section{Introduction}

Crystalline polymers can appear as hierarchical structures ranging from crystal lattices to spherulites. The hierarchical structures in a polymer object are affected in particular by tacticity, molecular weight distribution, and manufacturing processes. X-ray scattering provides a powerful tool to characterize these hierarchical structures [1,2]. Using a high-brilliance X-ray beam available at synchrotron radiation facilities, it is possible to trace scattering patterns on a sub-second-order time resolution, thus providing information on crystalline structure deformation dynamics during isothermal crystallization $[3,4]$, stretching $[5,6]$ and spinning processes $[7,8]$. On the other hand, X-ray scattering provide only generalized structural information that is averaged over the area irradiated with the X-ray beam, the diameter of which is on a micrometer-scale using the highly advanced microbeams available at synchrotron radiation facilities $[9,10]$. Polymer crystals are often heterogeneous, making it necessary to use real-space imaging to create a full picture of polymer objects. Atomic force microscopy (AFM), which provides topographic images on a nanometer-scale resolution, is a promising development for this purpose. At present, only a few studies have used this technique to observe polymer chains and fold-chain lamellae [11-16]. By using these techniques complementarily, there is great potential to research polymer structures in detail.

Zuo et al. [17] revealed structural changes in isotactic polypropylene (iPP) sheets uniaxially stretched to different extents at different temperatures by combining small-angle X-ray scattering (SAXS), wide-angle X-ray scattering (WAXS), and AFM: spherulites transformed into fibrils along the stretched direction. Moreover, by analyzing in-situ X-ray scattering patterns, they found deformation of the crystal and amorphous phases during uniaxial tensile deformation at both low and high temperatures.

Recent developments in AFM technology have been focused on high-resolution imaging of soft materials. Mullin et al. [18,19] observed polyethylene single chains in air by tapping them with T-shape cantilevers and measuring their torsional oscillation. Fukuma et al. [20] developed 
low-noise sensing of cantilever deflection in frequency-modulation AFM (FM-AFM), and imaged mica in water at a true atomic resolution. Force sensing in the frequency-modulation scheme enables imaging scans with limited tip-surface force and hence less invasive scans over soft objects. A number of soft materials have been imaged with liquid-compatible FM-AFM to date, including crystalline p-nitroaniline [21], rubrene [22], lysozyme [23], dodecanol adsorbed on graphite [24], hydrophilic thiolate monolayers on gold [25,26], lipid bilayers [27], purple membranes [28], peptide nanotubes [29], antibodies [30], and DNA [31]. As synthetic polymers, commercial sheets of polyethylene [32] and polypropylene [33] have also been imaged in liquids. In these previous works, the molecular-resolution images were obtained without using a Fourier filter. Therefore, in this study, the aim was to observe how the single chains in uniaxially stretched iPP sheets are imaged by liquid-compatible FM-AFM and compare the observations with X-ray scattering results.

\section{Experimental section}

Isotactic polypropylene (iPP) sheets were prepared by pressing a commercial iPP product (Sigma-Aldrich; number average molecular weight: 67000 weight average molecular weight: 250000). Purchased pellets were melted at $473 \mathrm{~K}$ for 5 min and pressed $10 \mathrm{MPa}$ using a hot press, then quenched at $293 \mathrm{~K}$. The prepared sheets were mounted between two clamps of a tensile instrument and uniaxially deformed while measuring the load. The engineering stress $\sigma$ is the load divided by the initial cross section, and the strain $\varepsilon$ is defined $\varepsilon=100\left(l-l_{0}\right) / l_{0}$ with $l_{0}$ and $l$ as the initial and final lengths of the sample, respectively. The initial length $l_{0}$ was $12 \mathrm{~mm}$ in this study. Uniaxial stretching measurements were carried out at $393 \mathrm{~K}$ with a stretching rate of $10 \%$ per min. In-situ SAXS and WAXS measurements were performed at the BL03XU of SPring-8 [34]. The radiating X-ray was monochromatized to be of a 0.1-nm wavelength and was introduced to the stretching film. The azimuth of the incident beam was perpendicular to the stretched direction. Small-angle scattering patterns were detected with an X-ray image intensifier with a cooled CCD, 
whereas wide-angle scattering patterns were collected on an X-ray flat-panel imager. The acquisition time was $2 \mathrm{~s}$ per frame.

The topography of the iPP sheets stretched by $0 \%$, $200 \%$, or $1500 \%$ was first observed in air at room temperature using a commercial AFM (Bruker, Multimode) operated in tapping mode using non-coated silicon cantilevers (Bruker, NCHV). Amorphous layers left on the press sheet ( $\varepsilon=0 \%)$ were stripped off by chemical etching [35]. The etching solution was prepared by dissolving $\mathrm{KMnO}_{4}(280 \mathrm{mg})$ in concentrated sulfuric acid $(12 \mathrm{ml})$ and carefully adding phosphoric acid (18 ml). The iPP sheets were immersed in the solution for 2 hours at room temperature, then transferred to a solution of dilute sulfuric acid (water $12 \mathrm{ml}+$ concentrated sulfuric acid $18 \mathrm{ml}$ ) and hydrogen peroxide (30 w/v\%) for 30 minutes each, washed with distilled water, and dried in air. The stretched iPP sheets ( $\varepsilon=200$ and $1500 \%$ ) were imaged without chemical etching.

The iPP sheets stretched by $200 \%$ or $1500 \%$ were then further imaged in phenyloctane liquid using another AFM (Shimadzu, SPM9600) modified for highly sensitive frequency-modulation sensing of tip-surface force. Silicon cantilevers backside-coated with aluminum (Nanosensors, NCH-R) were used. Phenyloctane was chosen for the imaging liquid since it does not dissolve iPP and has low volatility.

\section{Results and discussion}

Fig. 1 shows the stress-strain curve of an iPP sheet observed under uniaxial tensile deformation at $393 \mathrm{~K}$ with selected 2D WAXS and SAXS patterns. The stress was proportional to applied strains of up to $15 \%$ showing an elastic response of the stretched sheet. Beyond the yield point of 15\%, plastic deformation occurred with a gradual increase of stress until the sheet fractured at $1600 \%$. In the absence of strain, five Debye-Scherrer rings appeared in the WAXS pattern. An iPP polymer chain appears as a three-fold helix ( $3_{1}$ helix) with a repetition length of $0.65 \mathrm{~nm}$ along the helix axis [36]. Four polymorphs of crystalline iPP are known: the monoclinic $\alpha$-form (i.e., the dominant population), pseudo-hexagonal $\beta$-form, orthorhombic $\gamma$-form, and smectic forms [37]. According to 
the scattering vector lengths $\boldsymbol{q}$, the five rings observed in the WAXS pattern were assigned to diffractions of randomly oriented (110) at $\boldsymbol{q}=10.1 \mathrm{~nm}^{-1}$, (040) at $\boldsymbol{q}=12.0 \mathrm{~nm}^{-1}$, (130) at $\boldsymbol{q}=13.3 \mathrm{~nm}^{-1}$, (111) at $\boldsymbol{q}=15.2 \mathrm{~nm}^{-1}$ and $(13 \overline{1}) /(041)$ at $\boldsymbol{q}=15.6 \mathrm{~nm}^{-1}$ in the $\alpha$-form. (The lattice spacing of (131) and (041) of iPP $\alpha$-form are much the same.)

By applying strains beyond the yield point, a limited portion of each diffraction ring strengthened, resulting in an arc presentation. All (hk0) arcs, including (110), (040), and (130), transformed into diffraction peaks with further strain increases. The azimuth of the peaks was perpendicular to the stretched direction. In addition, the off-axis arc overlapped with (111) and (131) / (041). These results are consistent to earlier studies $[6,17,38]$ and suggest that plastic deformation induced crystallization in the $\alpha$-form with the c-axis parallel to the stretched direction. 


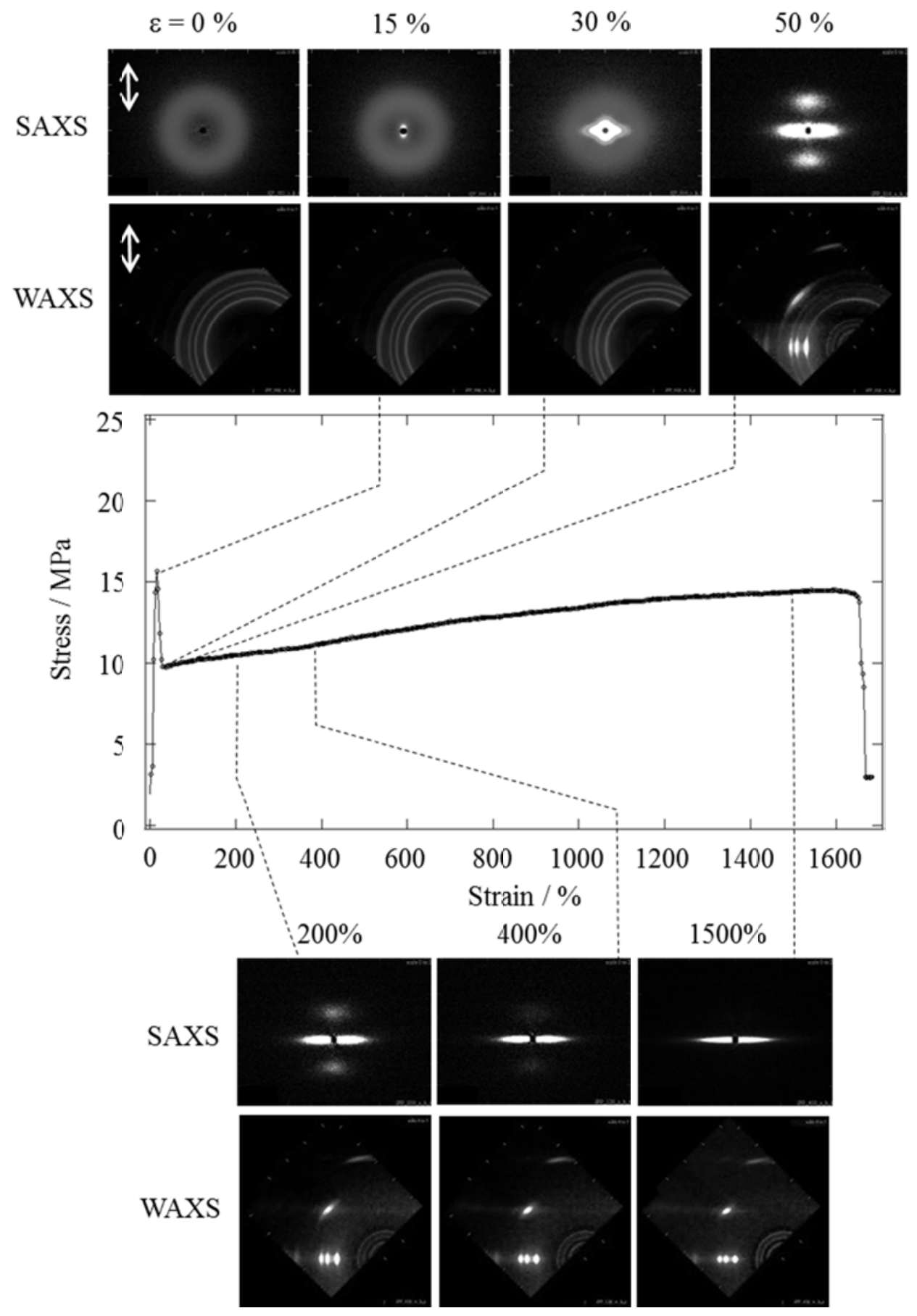

Fig. 1. The stress-strain curve of an iPP sheet. An iPP sheet was uniaxially stretched at $393 \mathrm{~K}$. The observed tensile stress is shown as a function of strain relative to the length of the unstretched sheet. Two-dimensional WAXS and SAXS patterns at different strains are inserted. The white arrows indicate the direction of stretching. 
In crystalline polymers, crystalline lamellae should coexist with amorphous domains. Crystalline areas are generally more densely packed than amorphous areas, and the electron density of the crystalline lamellae is slightly larger than that of the amorphous domains. X-ray diffraction in the domains of different electron densities contributes to an SAXS pattern. Accordingly, the average size of the domains can be estimated by analyzing the SAXS patterns. The observed SAXS patterns started with a broad isotropic ring, suggesting randomly oriented stacking of lamellae. A bright area appeared at the center with a strain of $30 \%$ and transformed into an equatorial streak with increasing strain until the sheet fracture. Two additional diffractions were generated at the top and bottom of the original ring. Both the WAXS and SAXS patterns suggested the presence of lamellar stack along the drawing direction. Fig. 2 shows the 1D SAXS profiles of the iPP sheets stretched by a) 200\% and b) $1500 \%$. The curves are integrated from the meridian region. A broad peak centered at $q=0.29$ $\mathrm{nm}^{-1}$ was observed at 200\%, which indicates a periodic structure with a center value of $21.7 \mathrm{~nm}$ along the direction of stretching. On the other hand, when stretched by $1500 \%$, there was no distinct peak. Further stretching resulted in crystallization from interlamellar coiled segments or fragmentation of crystalline lamella, and the periodic structure observed at 200\% disappeared. 


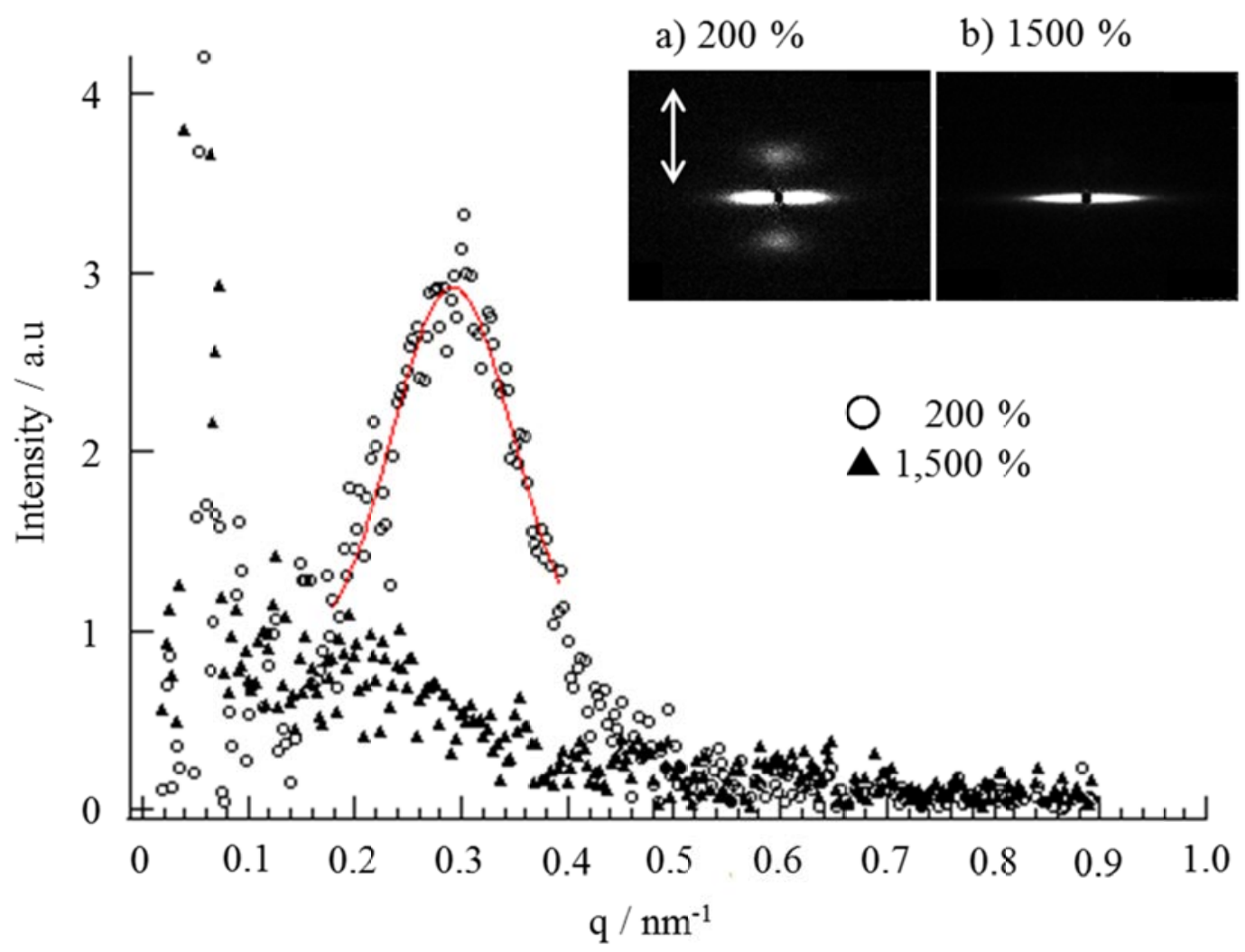

Fig. 2. 1D SAXS profiles of iPP stretched at $393 \mathrm{~K}$ : a) $200 \%$ and b) $1500 \%$ strain. The curves are integrated from the meridian region; corresponding 2D SAXS patterns are shown in the inset. The white arrows indicate the direction of stretching.

Fig. 3 shows the ex-situ AFM topography of another iPP sheet stretched at different strains. The topography of the sheets was observed in air at room temperature using a commercial AFM operated in tapping mode. In the topography of the unstretched sheet, which is shown in panel (a), radial structure and spherulites were recognized. The spherulites then transformed into fibrils when stretched, as shown in panels (b) and (c). The axis of the fibrils was parallel to the stretched direction. The SAXS patterns shown in Fig. 1 presented qualitatively different features at strains of 200 and $1500 \%$. The diffractions at the top and bottom of the ring were present at $200 \%$ and absent at $1500 \%$. However, the topography of the sheets stretched to these extents (shown in Fig. 3(b) and 3(c)) displayed similar features even when zoomed into small areas of the scan. This finding 
suggests that the spatial resolution of the microscope was not sufficient to recognize the topographic response related to the SAXS results. Hereafter, we employed FM-AFM to identify polymer-chain-scale features exposed on the iPP surfaces.


Fig. 3. Tapping-mode AFM topography of stretched iPP sheets. The strain of each sheet was (a) $0 \%$, (b) $200 \%$ and (3) $1500 \%$. The arrows indicate the direction of stretching.

Fig. 4 presents FM-AFM images of the stretched iPP sheet. When a solid object approaches a cantilever oscillating in mechanical resonance, the resonance frequency shifts due to the conservative force pushing or pulling the tip at the free end of the cantilever. The tip-surface distance is feedback-regulated to keep the shift of resonance frequency $(\Delta f)$ constant. In ordinary operations of FM-AFM, the object surface is traced in this manner to produce a constant frequency-shift topography. In imaging the stretched iPP sheets, we reduced the feedback gain for $\Delta f$ regulation and monitored the instantaneous $\Delta f$ relative to the $\Delta f$ set-point. When the tip passed over structures smaller than the fibrils protruding from the envelope, tip-surface distance reduced instantaneously. Hence, instantaneous $\Delta f$ positively shifted relative to the set-point. Mapping instantaneous $\Delta f$ as error from the set-point was a sensitive and convenient way to identify small corrugations embedded in the fibrils. However, in this type of topography, the absolute height was 
missed.

A frequency-shift error topography thereby obtained on the sheet stretched at $1500 \%$ is shown in Fig. 4. Two types of domains were recognized: one with crystalline lamellae, and the other assigned to amorphous iPP. Molecule-sized strings lay along the direction of stretching in the crystalline domains. The amorphous domains presented a structureless topography. The crystalline and amorphous domains appeared along the stretching direction. In earlier AFM studies [11-15] periodically folded chains were resolved on single-crystalline polymer objects. More complex features, crystalline domains embedded in amorphous domains, were identified with intra-domain structures in the frequency-shift error topography. The highly sensitive force detection in FM-AFM may have been helpful in resolving the complex features.

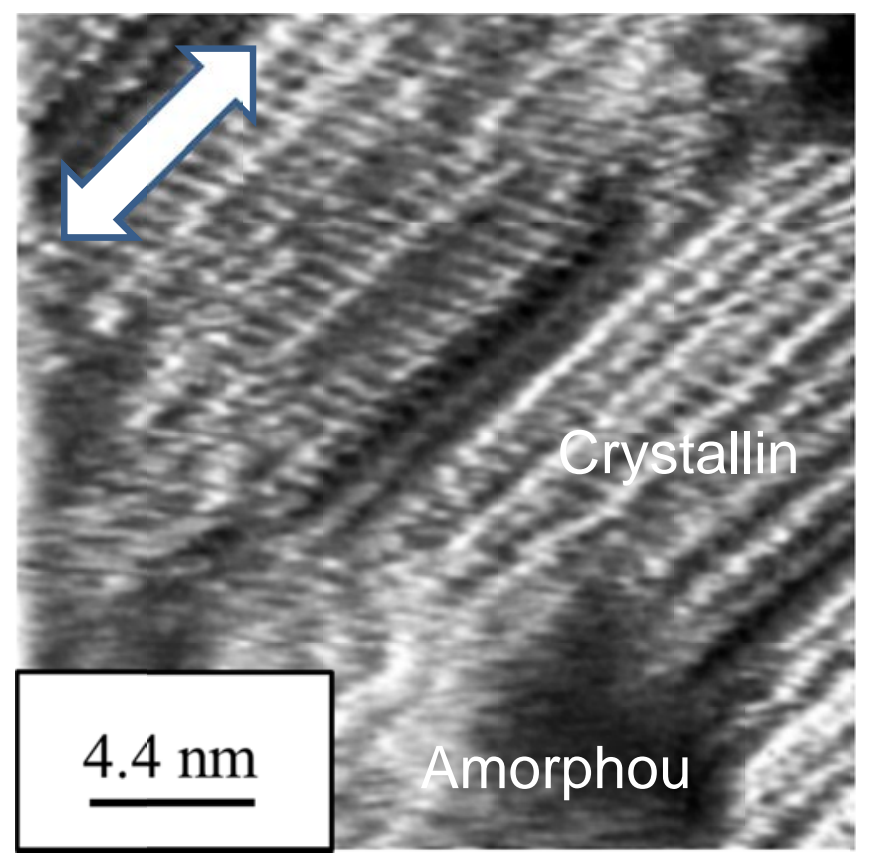

Fig. 4. A typical FM-AFM $\Delta$ f error image of the iPP sheet stretched at $1500 \%$.

Fig. 5(a)-(d) shows FM-AFM $\Delta$ f error images of the sheets stretched at 200\% with four different scan sizes. In large-area images (a) and (b), crystalline and amorphous domains were alternately present on individual fibrils. The typical length of the alternating patches was 10-60 nm, which is 
consistent with the reciprocal vectors of the diffraction peaks at the top and bottom of the SAXS pattern shown in Fig. 2. Intra-lamellae structures were resolved in small-area images (c) and (d). By further stretching at $1500 \%$, the size of the crystalline lamellae reduced to $10 \mathrm{~nm}$ or less, as depicted in large-area images (e) and (f). The large crystalline lamellae observed on the less-stretched sheet broke down in response to further stretching. It was evident in the series of $\Delta f$ error images that fragmentation of crystalline lamellae mainly occurred on the sheet stretched to $1500 \%$. Crystalline and amorphous domains were no longer well stacked or of uniform size, meaning the SAXS peaks were lost on this sheet, consistent with the findings in Fig. 2. Our FM-AFM imaging provided evidence in real-space supporting lamellae fragmentation by extensive stretching. 


\section{$200 \%$}
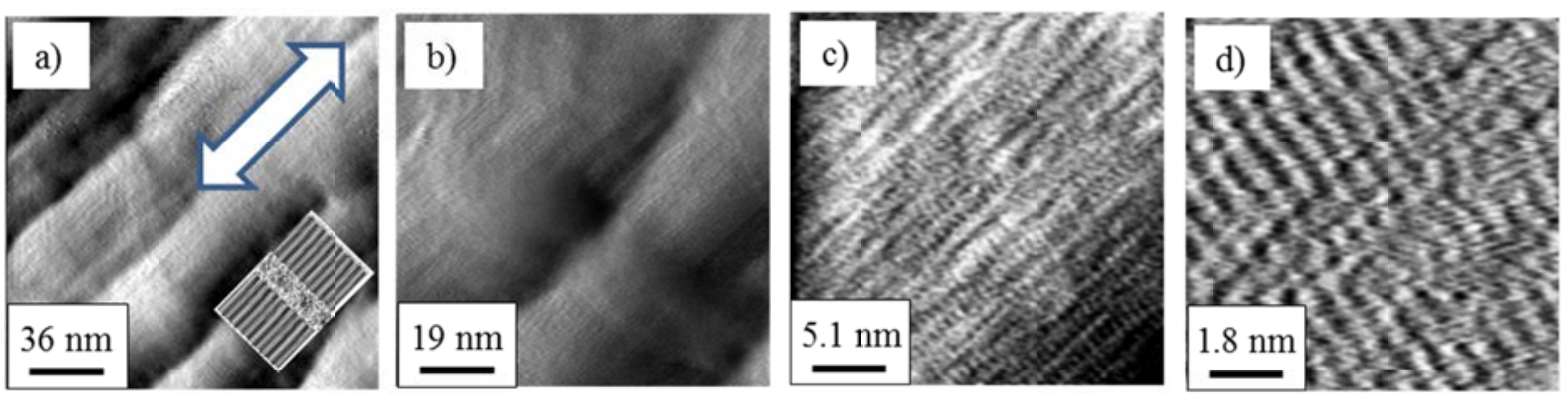

$1500 \%$
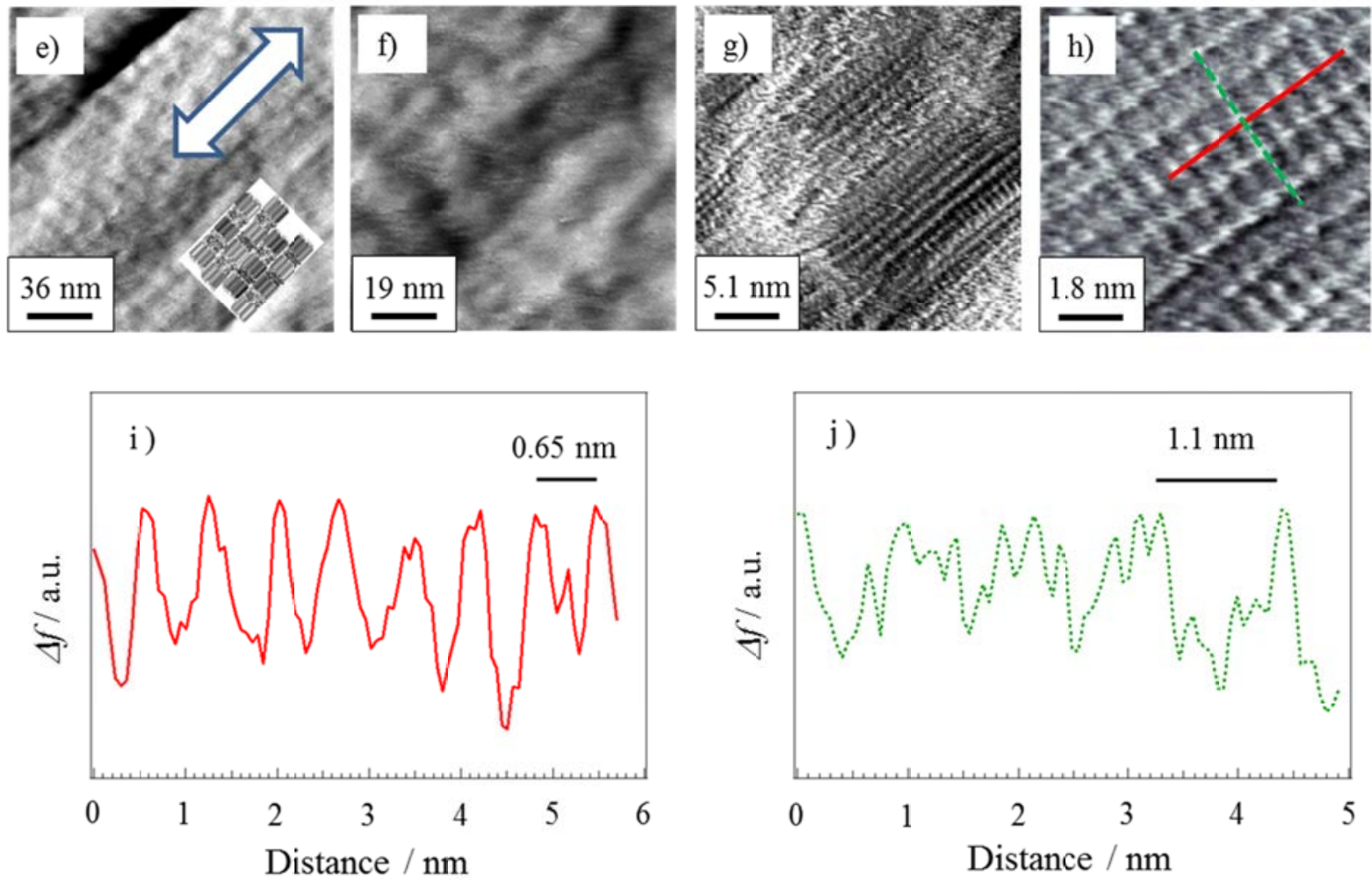

Fig. 5. FM-AFM $\Delta f$ error image of uniaxial stretching iPP: a) d) draw ratio $200 \%$, e) $\sim$ ) draw ratio 1500\%, i) and j) cross sectional profile of h). The arrows indicate the drawing direction. 
Molecule-sized strings in the crystalline domains on the sheet stretched 200\% are zoomed in on images (c) and (d). Similar strings with a better contrast were also seen on the sheet stretched $1500 \%$, as shown in images (g) and (h). Panel (i) shows $\Delta f$ error as a function of the lateral coordinate on the red solid line parallel to the stretched direction in image (h). The repetition length was $0.55-0.75 \mathrm{~nm}$, consistent with the helix pitch of crystalline iPP. This observation shows that the helix lay with the three-fold axis parallel to the stretched direction. Hence, the periodic protrusions were attributed to $\mathrm{CH}_{3}$ side-chain groups exposed on the surface.

Another cross section was made on the green dashed line shown perpendicular to the stretched direction, and its measurements are presented in panel (j). The repetition length in this direction was about $1.1 \mathrm{~nm}$. We ascribed the observed distance of neighboring strings to the periodic arrangement of helices placed on a (110) plane of crystalline iPP. Fig. 6 illustrates the structure of the crystalline $\alpha$ phase of iPP projected on a (001) plane. The $\alpha$ phase is monoclinic with lattice units and an angle of $\mathrm{a}=0.67 \mathrm{~nm}, \mathrm{~b}=2.10 \mathrm{~nm}, \mathrm{c}=0.65 \mathrm{~nm}$, and $\beta=99^{\circ} 8$ [37]. Left and right-handed helices are alternately placed. When the crystal is truncated on a (110) plane, left (or right) -handed helices protrude from the surface plane. The distance of neighboring, same-handed helices is $1.1 \mathrm{~nm}$, agreeing with the observed string distance. We therefore assume that the crystalline domain in image (h) was (110)-truncated. 


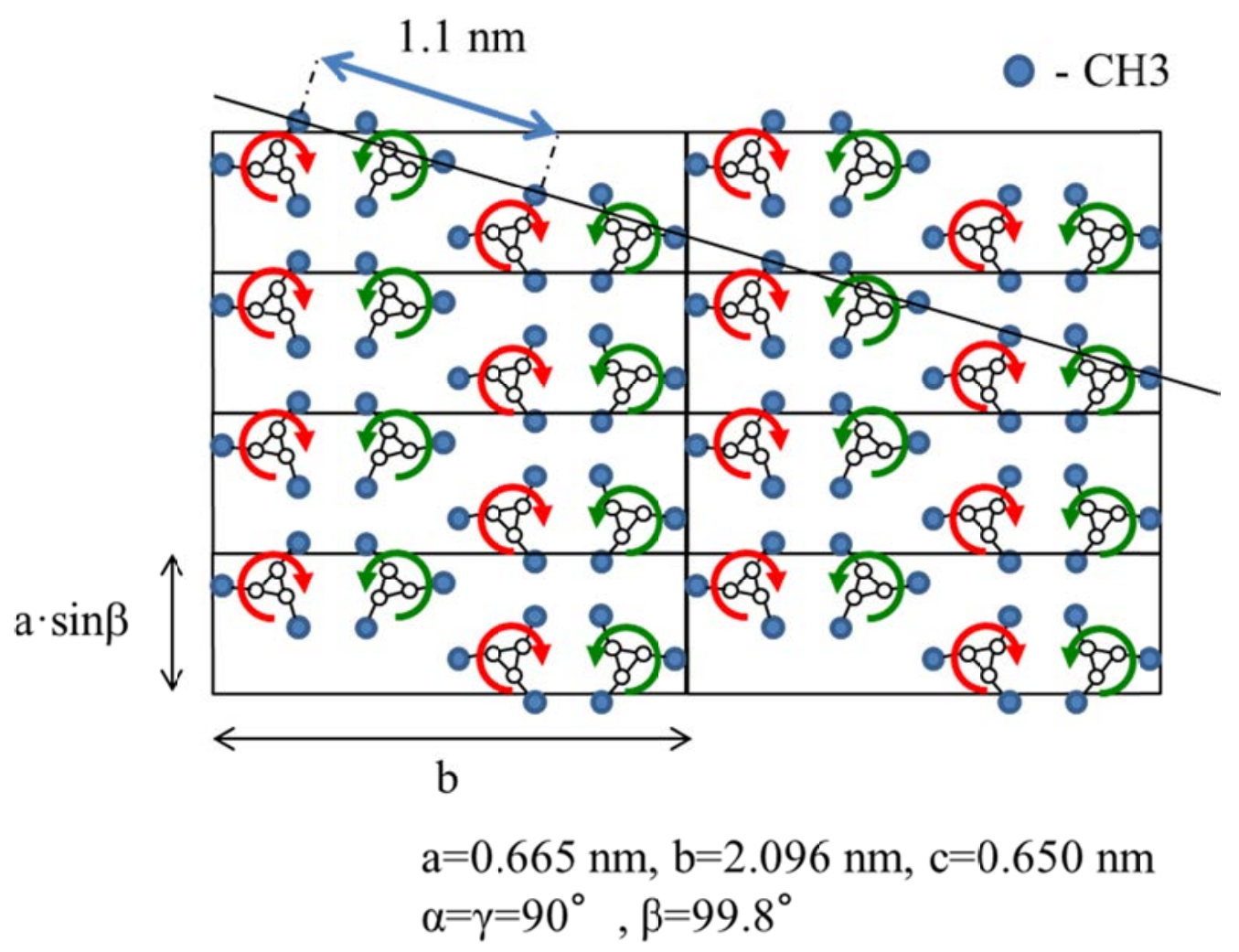

Fig. 6. Monoclinic $\alpha$ phase of iPP projected on a (001) plane. Two unit cells are shown, $a^{*}(=a$ * $\sin \beta$ ) axis vertical, b axis horizontal. Left (green arrows) - and right (red arrows) - handed helices are alternately placed.

\section{Conclusion}

The structures of isotactic polypropylene sheets uniaxially stretched up to $1500 \%$ were studied using advanced AFM operated in tapping and frequency-modulation (FM) modes, combined with X-ray scattering. Spherulites in the unstretched sheet transformed into fibrils when stretched. Crystalline and amorphous domains were identified in FM-AFM images of the fibrils. Crystalline domains of 10-60 nm lengths on the sheet stretched at 200\% underwent fragmentation into domains smaller than 10 -nm dimensions by further stretching to $1500 \%$. Three-fold helices with the axis parallel to the stretched direction were identified in the crystalline domains. Individual $\mathrm{CH}_{3}$ side-chains of three-fold helices were further recognized. The reciprocal-space features observed 
with small-angle and wide-angle X-ray scattering were consistent with the real-space images.

\section{Acknowledgement}

X-ray scattering measurements were done at the BL03XU of SPring-8 at the Japan Synchrotron

Radiation Research Institute under Proposal No. 2013A7218. The microscope operated in FM-mode was developed by the Advanced Measurement and Analysis Project of the Japan Science and Technology Agency in collaboration with Masahiro Ohta, Kazuyuki Watanabe, Mariachi Oyabu, Kei Kobayashi, Hirofumi Yamada, and Ryohei Kokawa. Financial support by a Grant-in-Aid for Scientific Research (B) with Grant Number 25286009 is acknowledged.

\section{References}

[1] G.S.Y.Yeh, R. Hosemann, J. Loboda-Čačković, H. Čačković, Annealing effects of polymers and their underlying molecular mechanisms, Polymer 17 (1976) 309-318.

[2] E.B. Bond, J.E. Spruiell, J.S. Lin, A WAXD/SAXS/DSC Study on the Melting Behavior of Ziegler-Natta and Metallocene Catalyzed Isotactic Polypropylene, J. Polym. Sci., Part B: Polym. Phys. 37 (1999) 3050-3064.

[3] Z. Wang, R.A. Phillips, B.S. Hsiao, Morphology Development during Isothermal Crystallization. I. Isotactic and Atactic Polypropylene Blends, J. Polym. Sci., Part B: Polym. Phys. 38 (2000) $2580-2590$

[4] K. Mita, H. Okumura, K. Kimura, T. Isaki, M. Takenaka, T. Kanaya, Simultaneous small- and wide-angle X-ray scattering studies on the crystallization dynamics of poly(4-methylpentene-1) from melt, Polym. J. 45 (2013) 79-86.

[5] S. Murakami, K. Senoo, S. Toki, S. Hohjiya, Structural development of natural rubber during uniaxial stretching by in situ wide angle X-ray diffraction using a synchrotron radiation, Polymer 43 (2002) 2117-2120. 
[6] T. Sakurai, Y. Nozue, T. Kasahara, K. Mizunuma, N. Yamaguchi, K. Tashiro, Y. Amemiya, Structural deformation behavior of isotactic polypropylene with different molecular characteristics during hot drawing process, Polymer 46 (2005) 8846-8858.

[7] R. Kolb, S. Seifert, N. Stribeck, H.G. Zachmann, Simultaneous measurements of small- and wide-angle X-ray scattering during low speed spinning of poly(propylene) using synchrotron radiation, Polymer 41 (2000) 1497-1505.

[8] S. Ran, X. Zong, D. Fang, B.S. Hsiao, B. Chu, R.A. Phillips, Structural and Morphological Studies of Isotactic Polypropylene Fibers during Heat/Draw Deformation by in-Situ Synchrotron SAXS/WAXD, Macromolecules 34 (2001) 2569-2578.

[9] R. Ishige, T. Shinohara, K.L. White, A. Meskini, M. Raihane, A. Taakahara, B. Ameduri, Unique Difference in Transition Temperature of Two Similar Fluorinated Side Chain Polymers Forming Hexatic Smectic Phase: Poly\{2-(perfluorooctyl)ethyl acrylate $\}$ and Poly\{2-(perfluorooctyl)ethyl vinyl ether\}. Macromolecules 47 (2014) 3860-3870.

[10] Y. Takanishi, H. Yao, T. Fukasawa, K. Ema, Y. Ohtsuka, Y. Takahashi, J. Yamamoto, H. Takezoe, A. Iida, Local Orientational Analysis of Helical Filaments and Nematic Director in a Nanoscale Phase Separation Composed of Rod-Like and Bent-Core Liquid Crystals Using Small- and Wide-Angle X-ray Microbeam Scattering, J. Phys. Chem. B 118 (2014) 3998-4004.

[11] O. Marti, H.O. Ribi, B. Drake, T.R. Albrecht, C.F. Quate, P.K. Hamsma, Atomic Force Microscopy of an Organic Monolayer, Science 239 (1988) 50-52.

[12] D. Snétivy, G.J. Vancso, Selective Visualization of Atoms in Extended-Chain Crystals of Oriented Poly(oxmethylene) by Atomic Force Microscopy, Macromolecules 25 (1992) $3320-3322$.

[13] S.N. Magonov, S.S. Sheiko, R.A.C. Deblieck, M. Möller, Atomic Force Microscopy of Gel-Draw Ultrahigh Molecular Weight Polyethylene, Macromolecules 26 (1993) 1380-1386.

[14] D. Snétivy, G.J. Vancso, Atomic force microscopy of polymer crystals: 7. Chain packing, disorder and imaging of methyl groups in oriented isotactic polypropylene. Polymer 35 (1994) 
461-467.

[15] W. Stocker, M. Schumacher, S. Graff, J. Lang, J.C. Wittmann, A. J. Lovinger, B. Lotz, Direct Observation of Right and Left Helical Hands of Syndiotactic Polypropylene by Atomic Force Microscopy, Macromolecules 27 (1994) 6948-6955.

[16] T. Kajiyama, I. Ohki, A. Takahara, Surface Morphology and Frictional Property of Polyethylene Single Crystals Studied by Scanning Force Microscopy, Macromolecules 28 (1995) 4768-4770.

[17] F. Zuo, J.K. Keum, X. Chen, B.S. Hsiao, H. Chen, S. Lai, R. Wevers, J. Li, The role of interlamellar chain entanglement in deformation-induced structure changes during uniaxial stretching of isotactic polypropylene, Polymer 48 (2007) 6867-6880.

[18] N. Mullin, C. Vasilev, J.D. Tucker, C.N. Hunter, C.H.M. Weber, J.K. Hobbs, “Torsional tapping” atomic force microscopy using T-shaped cantilevers. Appl. Phys. Lett. 94 (2009) 173109.

[19] N. Mullin, J.K. Hobbs, Direct Imaging of Polypropylene Films at Single-Chain Resolution with Torsional Tapping Atomic Force Microscopy. Phys. Rev. Lett. 107 (2011) 197801.

[20] T. Fukuma, K. Kobayashi, K. Matsushige, H.Yamada, True atomic resolution in liquid by frequency-modulation atomic force microscopy, Appl. Phys. Lett. 87 (2005) 034101.

[21] R. Nishioka, T. Hiasa, K. Kimura, H. Onishi, Specific Hydration on p-Nitroaniline Crystal Studied by Atomic Force Microscopy, J. Phys. Chem. C 117 (2013) 2939-2943.

[22] Y. Yokota, H. Hara, T. Harada, A. Imanishi, T. Uemura, J. Takeya, K. Fukui, Structural investigation of ionic liquid/rubrene single crystal interfaces by using frequency-modulation atomic force microscopy, Chem. Commum. 49 (2013) 10596-10598.

[23] K. Nagashima, M. Abe, S. Morita, N. Oyabu, K. Kobayashi, H. Yamada, M. Ohta, R. Kokawa, R. Murai, H. Matsumura, H. Adachi, K. Takano, S. Murakami, T. Inoue, Y. Mori, Molecular resolution investigation of tetragonal lysozyme (110) face in liquid by frequency-modulation atomic force microscopy. J. Vac. Sci. Technol. B 28 (2010) C4C11-C4C14. 
[24] W. Hofbauer, R. J. Ho, Hairulnizam R., N. N. Gosvami, S. J. O’Shea, Crystalline structure and squeeze-out dissipation of liquid solvation layers observed by small-amplitude dynamic AFM, Phys. Rev. B 80 (2009) 134104.

[25] T. Hiasa, K. Kimura, H. Onishi, Hydration of hydrophilic thiolate monolayers visualized by atomic force microscopy, Phys. Chem. Chem. Phys. 14 (2012) 8419-8424.

[26] T. Hiasa, H. Onishi, Mercaptohexanol assembled on gold: FM-AFM imaging in water, Colloids Surf., A 441 (2014) 149-154.

[27] T. Fukuma, M.J. Higgins, S.P. Jarvis, Direct Imaging of Lipid-Ion Network Formation under Physiological Conditions by Frequency Modulation Atomic Force Microscopy. Phys. Rev. Lett. 2007, 98, 106101.

[28] K. Kimura, S. Ido, N. Oyabu, K. Kobayashi, Y. Hirata, T. Imai, H. Yamada, Visualizing water molecule distribution by atomic force microscopy. J. Chem. Phys. 2010, 132, 194705.

[29] T. Sugihara, I. Hayashi, H. Onishi, K. Kimura, A. Tamura, Sub-nanometer-resolution imaging of peptide nanotubes in water using frequency modulation atomic force microscopy. Chemical Physics 416 (2013) 74-77.

[30] S. Ido, H. Kimiya, K. Kobayashi, H. Kominami, K. Matsushige, H. Yamada, Immunoactive two-dimensional self-assembly of monoclonal antibodies in aqueous solution revealed by atomic force microscopy. Nature Materails 13 (2014) 264-270.

[31] S. Ido, K. Kimura, N. Oyabu, K. Kobayashi, M. Tsukada, K. Matsushige, H. Yamada, Beyond the Helix Pitch: Direct Visualization of Native DNA in Aqueous Solution. ACS Nano 7 (2013) $1817-1822$.

[32] T. Hiasa, T. Sugihara, K. Kimura, H. Onishi, FM-AFM imaging of a commercial polyethylene film immersed in n-dodecane. J. Phys. Condens. Matter. 24 (2012) 084011.

[33] K. Suzuki, S. Kitamura, S. Tanaka, K. Kobayashi, H. Yamada, Development of High-Resolution Imaging of Solid-Liquid Interface by Frequency Modulation Atomic Force Microscopy. Jpn. J. Appl. Phys. 49 (2010) 08LB12. 
[34] H. Masunaga, H. Ogawa, T. Takano, S. Sasaki, S. Goto, T. Tanaka, T. Seike, S. Takahashi, K. Takeshita, N. Nariyama, H. Ohashi, T. Ohata, Y. Furukawa, T. Matsushita, Y. Ishizawa, N. Yagi, M. Tanaka, H. Kitamura, K. Sakurai, K. Tashiro, A. Takahara, Y. Amamiya, K. Horie, M. Takenaka, T. Kanaya, H. Jinnai, H. Okuda, I. Akiba, I. Takahashi, K. Yamamoto, M. Hikosaka, S. Sakurai, Y. Shinohara, A. Okada, Y. Sugihara, Multipurpose soft-material SAXS/WAXS/GISAXS beamline at SPring-8, Polym. J. 43 (2011) 471-477.

[35] M. Kuriyagawa, K. Nitta, Structural explanation on natural draw ratio of metallocene-catalyzed high density polyethylene, Polymer 52 (2011) 3469-3477.

[36] G. Natta, P. Corradini, Structure and Properties of Isotactic Polypropylene, del Nuovo Cimento XV (1960) 40-51.

[37] B. Lotz, J.C. Wittmann, A.J. Lovinger, Structure and morphology of poly(propylenes): a molecular analysis, Polymer 37 (1996) 4979-4992.

[38] F. Auriemma, C. De Rosa, Stretching Isotactic Polypropylene: From “cross- $\beta$ ” to Crosshatches, from $\gamma$ Form to $\alpha$ Form, Macromolecules 39 (2006) 7635-7647. 